\title{
A hidden target
}

Activation of the epidermal growth factor receptor (EGFR) is a common feature of many cancers, making it an attractive therapeutic target. Although agents that target EGFR have shown some promise, their use is limited owing to the side effects

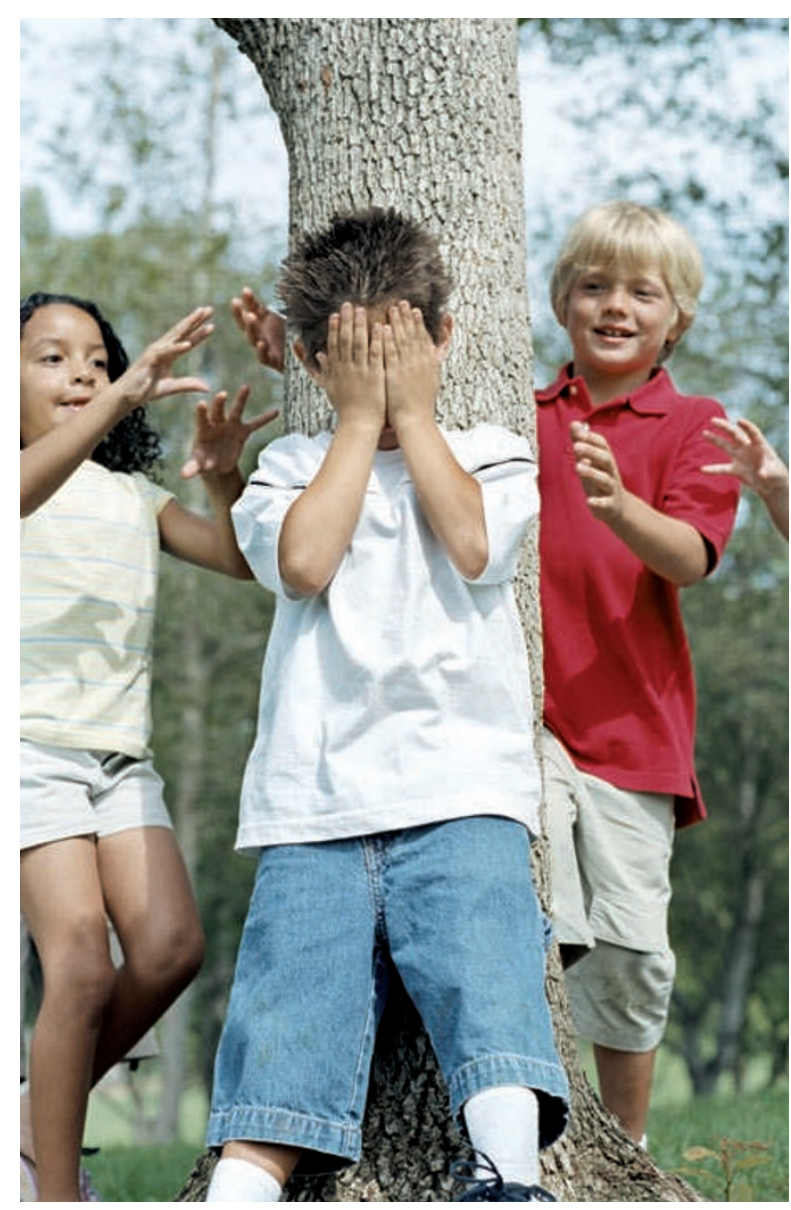

BANANASTOCK caused by interference with EGFR expressed on normal cells. A recent study suggests that it may be possible to create antibodies that specifically recognize tumour-associated EGFR by targeting a cryptic epitope.

Lloyd Old and colleagues previously generated a panel of monoclonal antibodies against a truncated version of EGFR (D2-7EGFR) that is associated with glioma. They identified two antibodies that can also bind overexpressed wild-type EGFR (wtEGFR) on cancer cells, but not wtEGFR expressed at endogenous levels on normal cells. The mechanism underlying this differential binding was unclear. To address this, Terrance Johns' team tested the anti-tumour activity of the two antibodies using nude mice xenografted with U87MG cells that express the D2-7EGFR mutant. The anti-EGFR antibodies resulted in a reduction in tumour volume. Significantly, xenografts from DU145 prostate cells, which contain an amplified transforming growth factor- $\alpha$ (TGFA) gene that leads to autocrine stimulation of wtEGFR, showed reduced tumour growth to a greater extent than the U87MG xenografts when treated with these antibodies.

What are the molecular interactions that lead to the specificity of these anti-EGFRs? The authors determined the crystal structures of the antibodies bound to their EGFR epitope, a short cysteine loop of the extracellular EGFR domain. Although this epitope adopts a similar conformation in wtEGFR, steric hindrance prevents the antiEGFRs from binding it. The authors identified a disulphide bond between two cysteine residues preceding the epitope that constrains the binding site. They reasoned that, if this bond were broken, the anti-EGFRs could bind to their epitope. In a proof-ofconcept experiment, they created an EGFR mutant with the two cysteines mutated to alanines. This resulted in exposure of the cryptic epitope and binding of the antibodies.

Why do these anti-EGFRs only bind to wtEGFR on cancer cells? One possibility is that cancer cells might express a misfolded form of the EGFR with a broken disulphide bond. However, the authors suggest that a more likely explanation is that, during EGFR activation, the receptor undergoes a conformational shift that exposes the cryptic epitope. Therefore, the antibodies can specifically bind to cancer cells with activated EGFRs. The results of this study indicate that designing antibodies to other misfolded or inappropriately activated receptors may be of benefit in cancer therapy.

Meera Swami

ORIGINAL RESEARCH PAPER Garrett, T. P. J. et al. Antibodies specifically targeting a locally misfolded region of tumor associated EGFR.

Proc. Natl Acad. Sci. USA 16 Mar 2009 (doi:10.1073/pnas.0811559106) 